\title{
Globally Coupled Parametrically Forced Logistic Maps
}

\author{
Hironori Kumeno \\ Dept. of Electrical and Electronic Eng., \\ Tokushima University, \\ Tokushima, 770-8506 JAPAN \\ Email: kumeno@ee.tokushima-u.ac.jp
}

\author{
Yoshifumi Nishio \\ Dept. of Electrical and Electronic Eng., \\ Tokushima University, \\ Tokushima, 770-8506 JAPAN \\ Email: nishio@ee.tokushima-u.ac.jp
}

\begin{abstract}
In this study, we propose a parametrically forced logistic map and investigate its bifurcation. By carrying out computer calculations, unique bifurcations from period to chaos are observed. Furthermore, we investigate synchronization phenomena in globally coupled logistic maps which involving parametric force and confirm various kinds of synchronization phenomena. In the case of two coupled maps, in-phase synchronization of two maps is observed. In the case of three coupled maps, synchronization states are changed with increasing coupling intensity from asynchronous, self-switching of synchronization, synchronization of two among the three maps, to synchronization of all the maps. Finally, we investigate the influences of phase difference of parametric forces on synchronization. The maps can not be synchronized completely if phase difference exists between two parametric forces of the maps.
\end{abstract}

\section{INTRODUCTION}

Synchronization is one of the fundamental phenomena in nature, and one of typical nonlinear phenomena. Therefore, studies on synchronization phenomena of coupled systems are extensively carried out in various fields, physics [1], biology [2], engineering and so on. However, the issues that should be investigated for synchronization remain in existence in spite of many researching. In particular, it is necessary to investigate synchronization phenomena in special conditions. There is parametric excitation that an amplitude of oscillation is increased by periodic varying of a parameter in some system. Parametric excitation circuit is one of resonant circuits, and it is important to investigate various nonlinear phenomena for future engineering applications. In a simple oscillator including parametric excitation, Ref. [3] reports that the almost periodic oscillation occurs if nonlinear inductor has saturation characteristic. Additionally the occurrence of chaos is referred in Refs. [4] and [5].

In the past we have investigated effects of parametric excitation of coupled van der Pol oscillators [6]. In this study, for more detailed investigation of the effect of parametric excitation on synchronization, we focus on a globally coupled system of simple one-dimensional maps. A typical scheme for global coupling is given by

$$
\begin{aligned}
& x_{i}(t+1)=(1-\varepsilon) f\left[x_{i}(t)\right]+\frac{\varepsilon}{N} \sum_{j=1}^{N} f\left[x_{j}(t)\right] \\
& (i=1,2, \cdots, N,)
\end{aligned}
$$

where $\varepsilon$ is the coupling intensity. The globally coupled maps are a scheme that an average number of all the maps affect each of the map, and similar to the system that we have studied using van der Pol oscillators. Hence, we investigate synchronization phenomena in a globally coupled system of one-dimensional maps which are forced by periodic parameter change. The one-dimensional map used in this study is a logistic map, since the map can be described by a simple discrete equation. The logistic map is a polynomial mapping, often cited as an archetypal example of how complex chaotic behavior can be arisen from very simple nonlinear dynamical equations. Mathematically, the logistic map is written as

$$
x(t+1)=\alpha x(t)(1-x(t)) .
$$

Typical one-parameter bifurcation diagram of the logistic map is obtained as shown in Fig. 1.

Firstly, we describe behaviors and bifurcations of the parametrically forced logistic map. Next, we investigate synchronization phenomena in the globally coupled parametrically forced logistic maps.

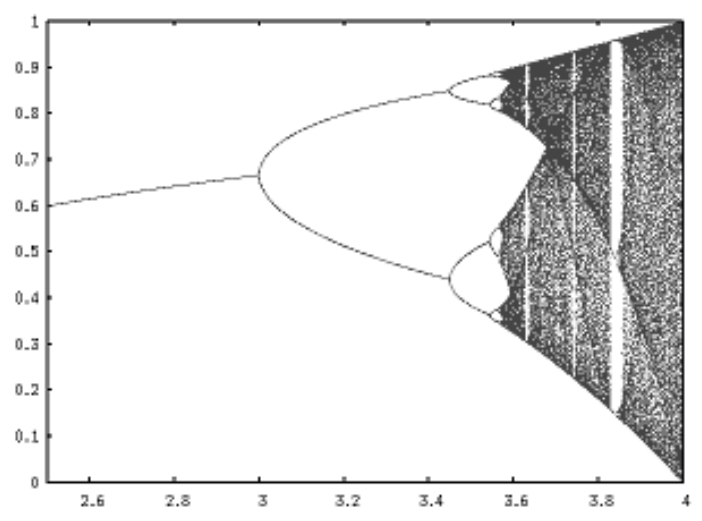

Fig. 1. One-parameter bifurcation diagram of logistic map.

\section{PARAMETRICALlY FORCED LOGISTIC MAP}

A parametrically forced logistic map used in this study is described as:

$$
x(t+1)=\alpha_{f}(t) x(t)(1-x(t)),
$$




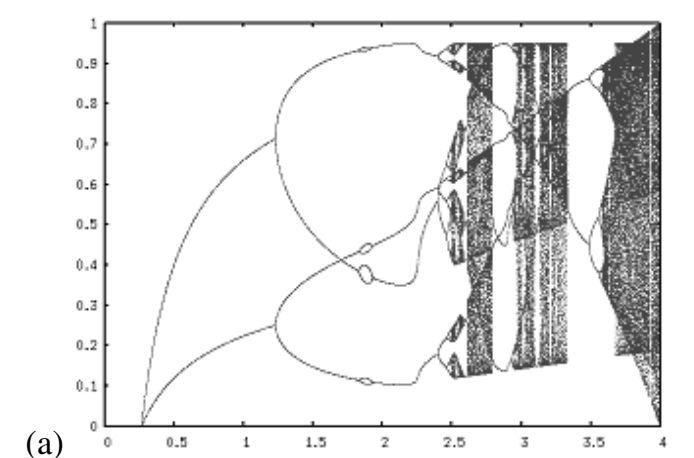

(a)

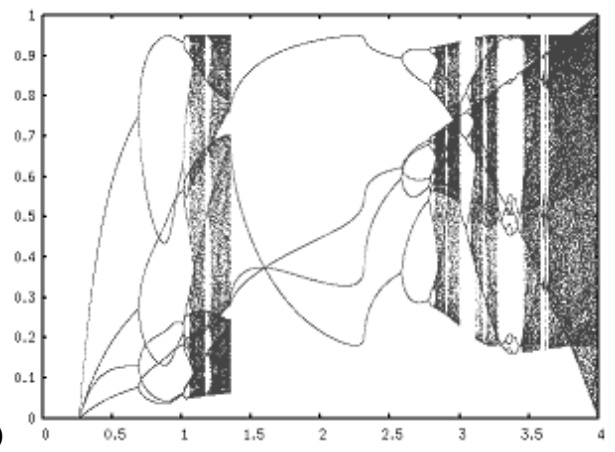

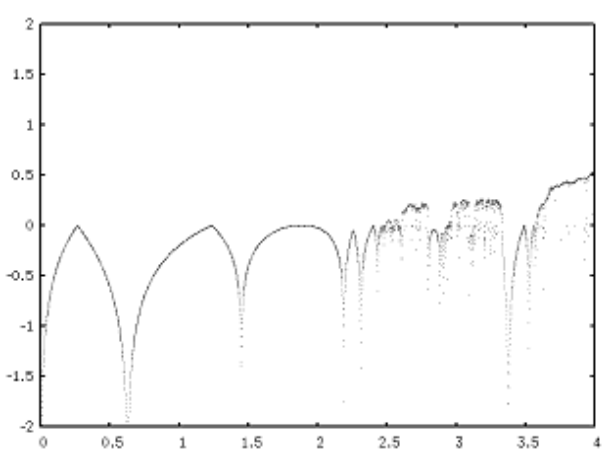

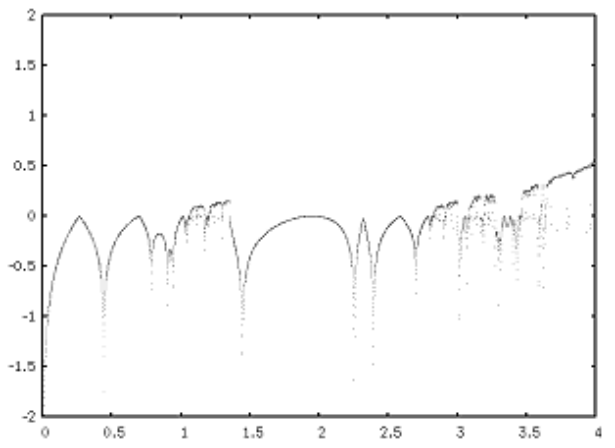

Fig. 2. One-parameter bifurcation diagrams (left) and the Lyapunov exponents (right) for $\alpha_{1}=3.8$. Horizontal axis: $\alpha_{2}$. (a) $\tau=1$. (b) $\tau=2$.

and

$$
\alpha_{f}(t)=\left\{\begin{array}{cc}
\alpha_{1}, & n\left(\tau_{1}+\tau_{2}\right)<t \leq n\left(\tau_{1}+\tau_{2}\right)+\tau_{1} \\
\alpha_{2}, & n\left(\tau_{1}+\tau_{2}\right)+\tau_{1}<t \leq(n+1)\left(\tau_{1}+\tau_{2}\right) \\
(n=1,2, \ldots)
\end{array}\right.
$$

where $\alpha_{f}(t)$ is a term of the parametric force and timevarying. The parametric force operation can be described as follows: in the time interval $n\left(\tau_{1}+\tau_{2}\right)<t \leq n\left(\tau_{1}+\tau_{2}\right)+\tau_{1}$, the system is driven by a parameter $\alpha_{1}$ during the duration $\tau_{1}$; while in the interval $n\left(\tau_{1}+\tau_{2}\right)+\tau_{1}<t \leq(n+1)\left(\tau_{1}+\tau_{2}\right)$, the system is driven by a parameter $\alpha_{2}$ during the duration $\tau_{2}$. Namely, in this system, two kinds of parameters are replaced alternately by the number of updates. Then, a parameter giving a periodic solution and a parameter giving another periodic solution can be combined. Of course, other combinations, for instance two parameters giving a periodic solution and a chaotic solution or two parameters giving two kinds of chaotic solutions, are possible. In this study, we assume $\tau_{1}=\tau_{2}=\tau$ for simplicity.

\section{BIFURCATION}

Before investigating synchronization phenomena in the globally coupled parametrically forced logistic maps, it is necessary to investigate behavior and bifurcation of one uncoupled parametrically forced logistic map. Figure 2 shows computer calculated results. Fixing a parameter $\alpha_{1}=3.8$ and $\tau=1$ and varying a parameter $\alpha_{2}$, the one-parameter bifurcation diagram and the Lyapunov exponents are obtained as shown in Fig. 2(a). Figure 2(b) shows the bifurcation diagram and the Lyapunov exponents for $\tau=2$. The different

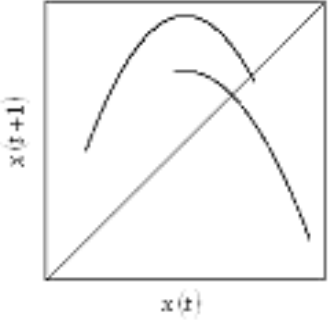

(a)

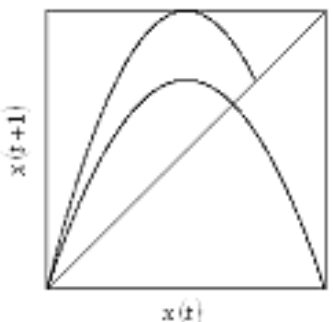

(c)

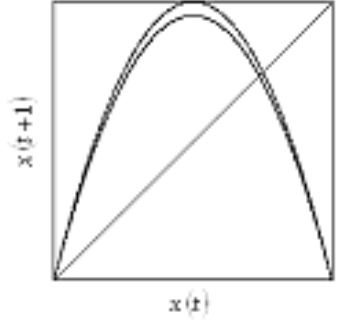

(b)

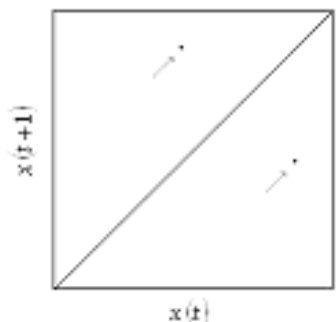

(d)
Fig. 3. Return maps of parametrically forced logistic maps for $\tau=1$. (a) $\alpha_{1}=3.0$ and $\alpha_{2}=3.8$. (b) $\alpha_{1}=3.8$ and $\alpha_{2}=4.0$. (c) $\alpha_{1}=3.0$ and $\alpha_{2}=4.0$. (d) $\alpha_{1}=3.5$ and $\alpha_{2}=4.0$.

types of bifurcation diagrams are obtained. From these figures, observations of periodic and chaotic attractors are confirmed.

Figure 3 show some examples of the return maps of the parametrically forced logistic maps. For the original logistic map, two-periodic solution is observed for $\alpha=3.0$. While, three-periodic solution is observed for $\alpha=3.8$. These two solutions are periodic, whereas in the logistic map involving parametric force, a solution is chaotic as shown in Fig. 3(a) 
when the parameters $\alpha_{1}$ and $\alpha_{2}$ are set 3.0 and 3.8. Namely, chaotic solution can be observed in the combination of two parameters that generate two kinds of periodic solutions.

\section{SYNCHRONIZATION}

In this section, we investigate synchronization phenomena by carrying out computer calculations for the cases of two and three maps. In the following computer calculations, the parameters are fixed as $\alpha_{1}=3.8, \alpha_{2}=4.0$ and $\tau=1$.

\section{A. Two maps case}

In this subsection, we consider the case of $N=2$, namely only two parametrically forced logistic maps are coupled. In this case, in-phase synchronization of two maps is observed. Figure 4 shows computer calculated results. As shown in the figures, with $\varepsilon$ increases, chaotic solutions obtained from two maps become to be synchronized. When $\varepsilon=0.450$, two chaotic attractors seem to be completely synchronized.

(a)
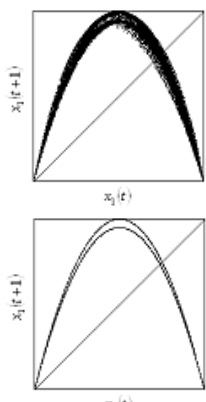

(b)
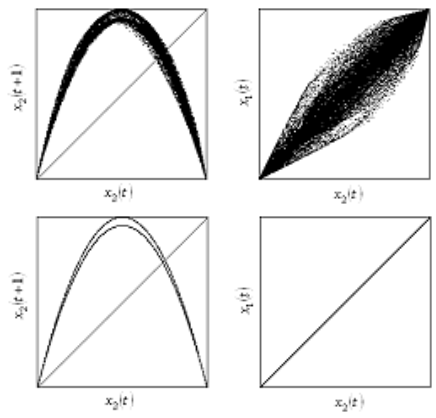

Fig. 4. In-phase synchronization of two maps for $\alpha_{1}=3.8, \alpha_{2}=4.0$ and $\tau=1$. (a) $\varepsilon=0.405$. (b) $\varepsilon=0.450$.

\section{B. Three maps case}

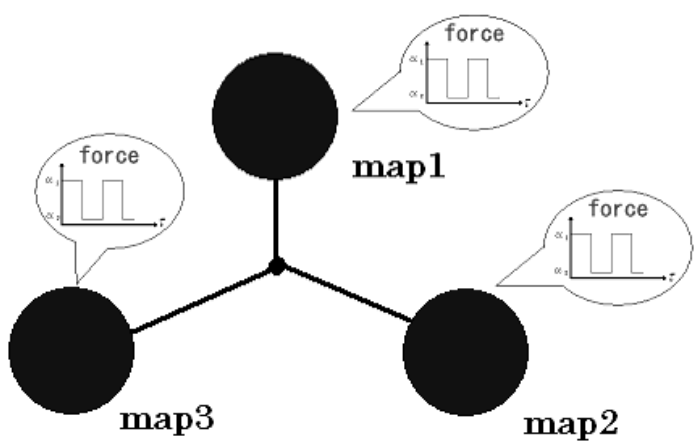

Fig. 5. Conceptual structure of three coupled parametrically forced logistic maps.

In this subsection, we consider the case of $N=3$. Figure 5 shows a conceptual structure of three coupled parametrically forced logistic maps. In this case, various kinds of synchronization phenomena are observed with increasing coupling intensity $\varepsilon$. Figure 6 shows examples of synchronization phenomena. In Fig. 6, upper figures show the return maps and lower figures show the phase differences between the maps.
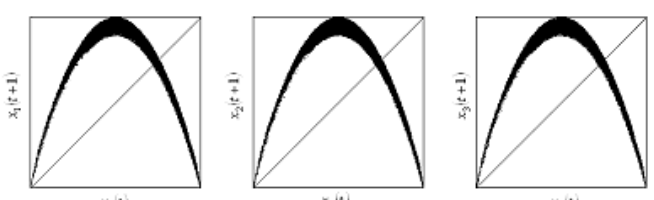

(a)
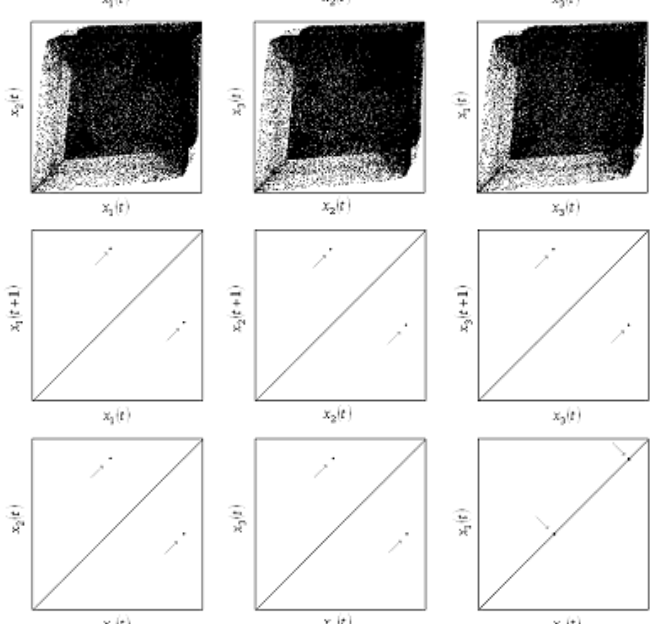

(b)
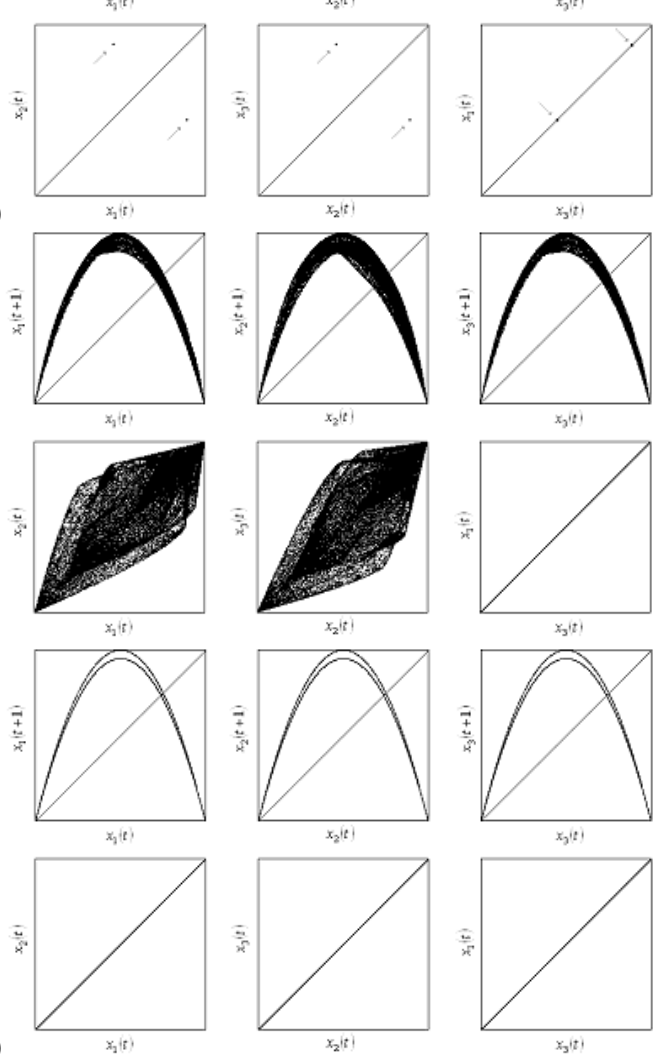

Fig. 6. Synchronization of three chaos. $\alpha_{1}=3.8, \alpha_{2}=4.0$ and $\tau=1$. (a) $\varepsilon=0.10$. (b) $\varepsilon=0.30$. (c) $\varepsilon=0.32$. (d) $\varepsilon=0.41$.

First, when the coupling parameter $\varepsilon$ is small, the maps are almost asynchronous as shown in Fig. 6(a). Increasing the coupling intensity, self-switching phenomenon of synchronization is observed. In this phenomenon, two of the three chaotic maps are synchronized. While, the combination of the two synchronized maps switches with time at random. As increasing the coupling intensity, synchronization of two of the three maps which generate periodic solutions is observed as shown in Fig. 6(b). In the figure, the two maps are synchronized, whereas the remain map seem to be synchronized with the others at the opposite-phase, since solutions of the maps 
are periodic. Moreover, as increasing the coupling intensity further, synchronization of two of the three maps which generate chaotic solutions is observed as shown in Fig. 6(c). In the figure, all return maps behave chaotic, and two maps, the left return map and the right return map, are synchronized. While, the shapes of the return maps are different from that of the uncoupled map. Finally, when the coupling intensity $\varepsilon$ is over 0.4 , synchronization of all the maps which generate chaotic solutions is observed as shown in Fig. 6(d). In the figure, all return maps are chaotic and have the same shapes as a return map of the uncoupled map.

\section{EFFECT OF PHASE DIFFERENCE}

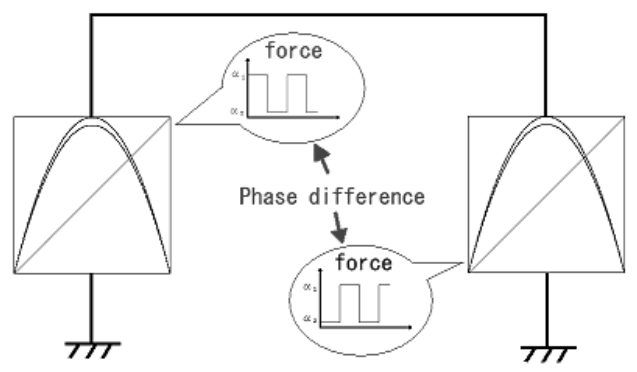

Fig. 7. Conceptual structure of Phase difference between parametric forces.

In this section, in order to investigate the synchronous influence of phase difference of parametric force, we focus on the two coupled chaotic maps whose parameters are $\alpha_{1}=3.8$, $\alpha_{2}=4.0$ and $\tau=6$. Figure 7 shows a conceptual structure of a phase difference between the parametric forces of the maps. In the above parameters, $\alpha_{f}(t)$ switches every six updates and the chaotic maps are synchronized if the parametric forces have no phase differences. With shifting the timing of the switch of $\alpha_{f}(t)$, we investigate synchronous influence of the phase difference. Figure 8 shows the computer calculated results. In the figures, two maps are synchronized if the phase difference does not exist between the two parametric forces of the maps (see Fig. 8(a)). Increasing the phase difference, the two maps become out of synchronization (see Figs. 8(b) and (c)). Additionally, if the phase difference exists between two parametric forces of the maps, no matter how strong coupling intensity increase, two maps can not be synchronized completely.

\section{CONCLUSION}

In this study, firstly, we proposed a parametrically forced logistic map and investigated its bifurcations. By carrying out computer calculations, unique bifurcations from period to chaos were observed. Furthermore, we investigated synchronization phenomena in the globally coupled parametrically forced logistic maps and observed various kinds of synchronization phenomena. In the two maps case, in-phase synchronization of two maps was observed. In the three (a)
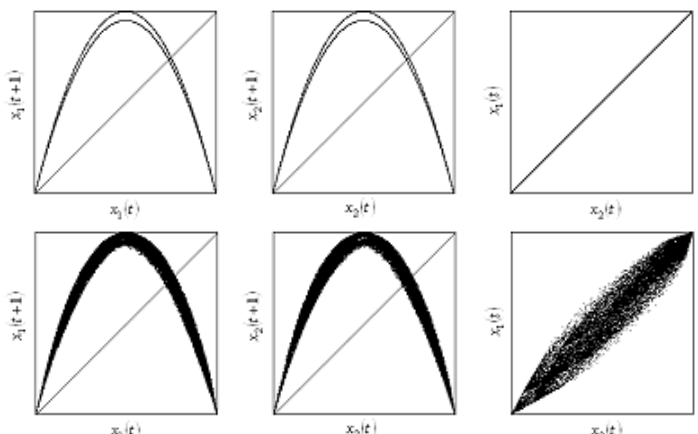

(b)
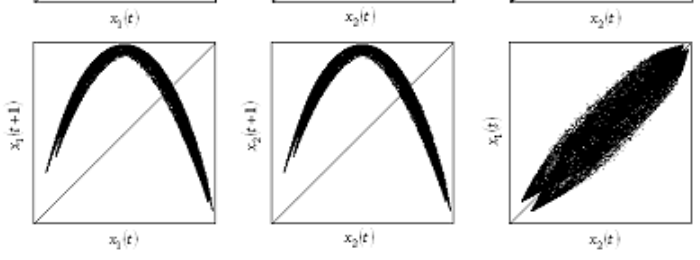

Fig. 8. Return maps and phase differences. $\alpha_{1}=3.8, \alpha_{2}=4.0$ and $\tau=6$. (a) phase difference $=0$. (b) phase difference $=4$. (b) phase difference $=6$.

maps case, synchronization states were changed with increasing coupling intensity from asynchronous, self-switching of synchronization, synchronization of two of the three maps to synchronization of all the maps. Finally, we investigated the influences of phase difference of parametric force on synchronization. We observed that if phase difference exists between the two parametric force of the maps, no matter how strong coupling intensity increase, two maps can not be synchronized completely.

\section{ACKNOWLEDGMENT}

This work was partly supported by Yazaki Memorial Foundation for Science and Technology.

\section{REFERENCES}

[1] I. Belykh, M. Hasler, M. Lauret and H. Nijmeijer, "Synchronization and graph topology," Int. J. Bifurcation and Chaos, vol.15, no.11, pp.34233433, Nov. 2005.

[2] J. Cosp, J. Madrenas, E. Alarcon, E. Vidal and G. Villar, "Synchronization of nonlinear electronic oscillators for neural computation," IEEE Trans. Neural Networks, vol.15, no.5, pp.1315-1327, Sep. 2004.

[3] C. Hayashi, "Nonlinear Oscillations in Physical Systems," Chap. 11, McGraw-Hill, New York (1964).

[4] C. Hayashi, M. Abe, K. Oshima and H. Kawakami, "The Method of Mapping As Applied To the Solution for Certain Types of Nonlinear Differential Equations," Proc. of the Ninth International Conference on Nonlinear Oscillations, pp. 1-8, 1981.

[5] M. Inoue, "A Method of Analysis for the Bifurcation of the Almost Periodic Oscillation and the Generation of Chaos in a Parametric Excitation Circuit," Trans. of IEICE, vol. J68-A, no. 7, pp. 621-626, 1985.

[6] H. Kumeno and Y. Nishio, "Synchronization Phenomena in Coupled Parametrically Excited van der Pol Oscillators," Proceedings of International Symposium on Nonlinear Theory and its Applications (NOLTA' 08), pp. 128- 131, Sep. 2008 